\title{
Phase based Indoor Real-Time Tracking of Mobile UHF RFID tags
}

\author{
Haishu Ma and Kesheng Wang \\ Department of Production and Quality Engineering \\ Norwegian University of Science and Technology \\ Trondheim, Norway \\ haishu.ma@ntnu.no
}

\begin{abstract}
Indoor real-time location system (RTLS) has attracted more and more attention due to the growing demands on location-based services (LBSs). Many localization algorithms have been developed, but most of them are either expensive to implement or suffering from low accuracy because of various impacts, e.g. Occlusion, multipath, and device heterogeneity. This paper presents a novel tracking method based on the measured phase values. Our method could provide real-time tracking of mobile RFID tags using commercial off the shelf(COTS) devices without comprising the accuracy. The basic idea to realize tracking is that the trajectory can be approximated by a series of line segments. Between two consecutive time steps, the tag's velocity can be assumed to be constant. The instant speed at every line segments can be estimated by differentials of the phase measurements. The starting point of the trajectory is calculated using received signal strength (RSSI) through least squares. Then the trajectory can be recursively inferred from the integral of velocity. The performance of our methods is studied in simulations and experimentally verified in our lab.
\end{abstract}

Keywords- RFID; tracking; phase differential; phase measurement; least squares

\section{INTRODUCTION}

Locations based services(LBSs) has become one of the most attractive fields as the rapid development of wireless sensor network(WSN). These networks have been widely deployed for smart home monitoring, asset tracking and rescue operation[1]. Global positioning system(GPS) is the most well known outdoor navigation system, but performs poorly in indoor environments due to the lack of line of sight transmission channel between satellites and an indoor receiver[2]. In order to meet the increasing demands on indoor positioning and navigation, great efforts have been devoted to developing the indoor real-time tracking system. Compared with existing solutions using laser scanners, cameras, or ultrasound, RFID has become a hot topic in recent years.

Radio frequency identification (RFID) is an emerging technology which uses wireless communication for automatic identification of objects. The basic components of an RFID system consist of tags and readers. The tag is attached to the object to be identified and located. Because of its lower cost and the potential to locate object with high accuracy, RFID has been widely adopted as an attractive technology in asset tracking, industrial automation, and healthcare system[3].
One of the most popular techniques in RFID localization is LANDMARC, which deploys reference tags at known positons and then estimate the target's position based on the k-nearest neighbor algorithm[4]. One assumption for this kind of methods is that the signal is propagating in a free space. However, RSSI is not a reliable indicator for position in a multipath environment especially when the tag is moving. Tag's orientation and antenna gain all will affect the RSSI measurements. Fingerprinting method has been acknowledged to be able to provide high localization accuracy. However fingerprinting requires the laborious manual collection of RSS values during the offline stage[5]. Moreover, it will suffer from device heterogeneity which will degrade its performance. Previous studies show that time of arrival(TOA) and time difference of arrival(TDOA) leads to more accurate location estimation. Nevertheless, these techniques require high-cost hardware to implement[6]. A more recent knowledge-based study proposes using inverse (synthetic aperture) SAR to locate moving RFID tags, which results in fine-grained localization with accuracy of $\mathrm{cm}$ level[7]. One premise of this method is that the velocity and trajectory of the tag is predefined.

Our method differentiates from SAR on that the tracking can be achieved without the velocity and trajectory being known in advance. The tracking begins with the initial position estimation based on the received signal strength using least squares. Then we estimate the instant velocity of the mobile tag through the differential of two consecutive phase measurements. Our approach is cost-efficient because only COTS devices are used without any modification of hardware.

The rest of the paper is organized as follows. The background of phase measurements is introduced in section II. We present the proposed approach in section III. Section IV gives the simulation results of our method. The performance of our method is tested in a real scenario in section V. Finally, section VI concludes our work.

\section{BACKGROUND}

Ultra-high frequency (UHF) RFID system communicates through backscatter radio link. Fig. 3 displays the backscatter communication between a reader and a tag. Passive RFID tag has no battery. Instead, they draw power from the reader, which transmits electromagnetic waves that induce a current in the tag's antenna. The RF signal transmitted by the reader is 
reflected off the tag, received back at the reader and processed to decode the data. The tag will reply the reader's query by changing the impedance on its antenna and modulates its data on the backscatter signals using ON_OFF keying[8].

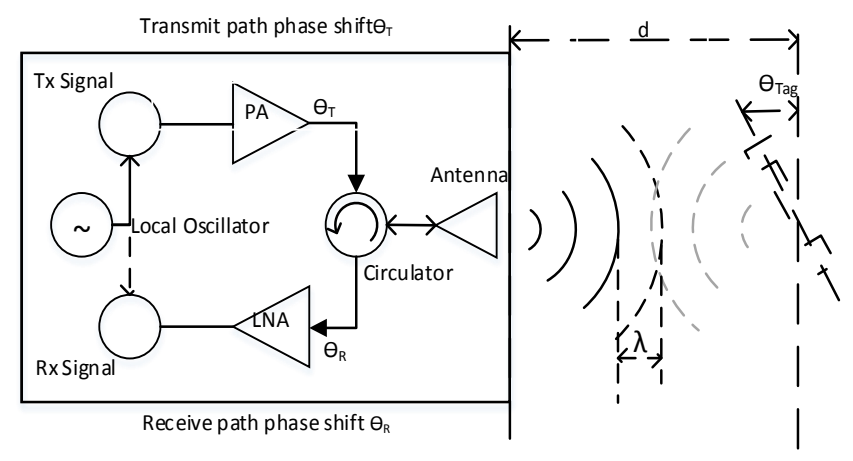

Fig. 1. Radio wave propagation between reader and tag

\section{A. RF phase}

Most COTS RFID reader can report phase value $\theta$, which is the phase shift between the transmitted and received signal. Whenever a tag is interrogated by a reader, phase report is generated. As illustrated in Fig. 3, d is the distance from tag to reader. The total propagation distance of RF signal from the reader to the tag and back again is $2 \mathrm{~d}$. However, reader's transmitter, receiver circuits, and tag's antenna will all contribute extra phase rotations, which make the measured phase not purely related with distance between reader and tag. The measured phase is a function of the wavelength $\lambda$ and total propagation distance $2 \mathrm{~d}[9]$. The formula can be express as:

$$
\theta=\left(\frac{2 \pi}{\lambda} \times 2 d+\theta_{T}+\theta_{R}+\theta_{T a g}\right) \bmod 2 \pi
$$

Where $\theta_{T}, \theta_{R}$ and $\theta_{T a g}$ are the additional phase rotations introduced by reader transmitter, receiver and tag respectively. They are what we call device heterogeneity.

\section{B. Trajectory approximation}

Suppose a mobile RFID tag is moving along an unknown track. Its position $\vec{r}(t)$ at time stamp $t$ is unpredictable, but its velocity $\vec{v}(t)$ can be measured. When the time interval is short enough, it can be assumed that the tag is moving along a linear line with constant speed.

The reader employed in our design is Sirit Infinity 610, which operates in the $860-960 \mathrm{MHz}$ band. An average read-out speed of the Sirit Infinity reader is 400 times per second. If there are four antennas connected to the same reader, the readout speed of each reader antenna is 100 times per second. Suppose the tag is moving at a speed of $5 \mathrm{~m} / \mathrm{s}$, the tag moves only $5 \mathrm{~cm}$ during the time interval. Usually the automated guide vehicle $(\mathrm{AGV})$ and forklift in the warehouse are moving under $5 \mathrm{~m} / \mathrm{s}$. Therefore it is reasonable for our assumption that the trajectory can be approximated by a series of uniform linear motion.

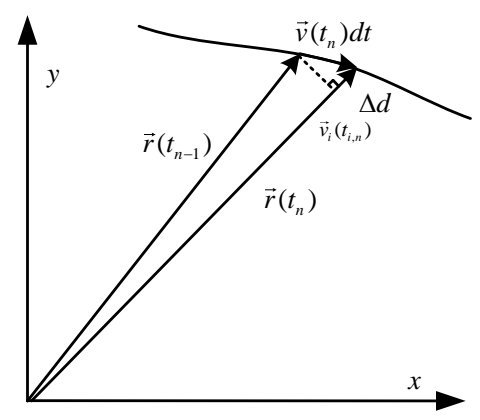

Fig. 2. Trajectory approximation with uniform linear motion

Accordingly, the tag's position $\vec{r}\left(t_{n}\right)$ at time stamp $t_{n}$ can be expressed as:

$$
\vec{r}\left(t_{n}\right)=\vec{r}\left(t_{n-1}\right)+\vec{v}\left(t_{n}\right) d t
$$

As shown in figure 2. As long as the initial position is known, the trajectory can be calculated recursively using the following equation:

$$
\vec{r}(t)=\vec{r}\left(t_{0}\right)+\int \vec{v}(t) d t
$$

\section{TRACKING APPROACH}

\section{A. Initial point estimation}

The starting point is estimated using received signal strength indicator (RSSI). Suppose $R_{i}$ is the measured RSSI by the $i^{\text {th }}$ reader antenna. Then based on the most commonly used Log-distance path loss model(LPDL), we obtain:

$$
R_{i}=P L_{0}+10 \alpha \log \left(d_{i}\right)+X_{\sigma}(d B)
$$

Where $P L_{0}$ is the reference path loss coefficient, $\alpha$ is the path loss exponent, and $d_{i}$ denotes the distance between the tag and the $i^{\text {th }}$ antenna. $X_{\sigma}(d B)$ is zero mean Gaussian random variable which is used to model the random characteristics of indoor radio signal propagation due to the various environmental factors such as multipath, obstruction, tag orientation. Assume the tag's initial positon is $\vec{r}\left(t_{0}\right)=\left(x_{0}, y_{0}\right)$ and the $i^{t h}$ antenna's position is $\vec{A}_{i}=\left(X_{i}, Y_{i}\right)$. The distances returned by the LPDL model can be related to the tag's position using the following expression:

$$
\left(x_{0}-X_{i}\right)^{2}+\left(y_{0}-Y_{i}\right)^{2}=d_{i}^{2}
$$

Assume we have $N$ reader antennas. We can construct $N$ equations in the same way. By subtracting $d_{1}^{2}$ from $d_{i}^{2}$ $(i=2,3, \ldots, N)$, the initial position can be calculated as:

$A \vec{x}=\vec{b}$
Where $A=2\left[\begin{array}{cc}\left(X_{2}-X_{1}\right) & \left(Y_{2}-Y_{1}\right) \\ \vdots & \vdots \\ \left(X_{N}-X_{1}\right) & \left(Y_{N}-Y_{1}\right)\end{array}\right], \vec{x}=\left[\begin{array}{c}x_{0} \\ y_{0}\end{array}\right]$ and 
$\vec{b}=\left[\begin{array}{c}\left(X_{2}^{2}-X_{1}^{2}\right)+\left(Y_{2}^{2}-Y_{1}^{2}\right)-\left(d_{2}^{2}-d_{1}^{2}\right) \\ \vdots \\ \left(X_{N}{ }^{2}-X_{1}^{2}\right)+\left(Y_{N}{ }^{2}-Y_{1}^{2}\right)-\left(d_{N}{ }^{2}-d_{1}^{2}\right)\end{array}\right]$.

By leveraging the linear least squares method, the initial position can be estimated.

\section{B. Instant velocity estimation}

As mentioned in section II, the length traveled by the tag is far less than half a wavelength. This feature allows us to estimate the tag's radical speed, which is the projection of the tag's instant velocity vector on the line of sight between the tag and the reader antenna. As illustrated in Fig.2, the tag's displacement $\Delta d$ can be calculated using (1):

$$
\Delta d=\left\{\begin{array}{c}
\frac{\varphi_{i}\left(t_{i, n+1}\right)-\varphi_{i}\left(t_{i, n}\right)}{4 \pi} \lambda,\left|\varphi_{i}\left(t_{i, n+1}\right)-\varphi_{i}\left(t_{i, n}\right)\right|<\pi \\
\frac{2 \pi-\varphi_{i}\left(t_{i, n}\right)+\varphi_{i}\left(t_{i, n+1}\right)}{4 \pi} \lambda, \varphi_{i}\left(t_{i, n+1}\right)-\varphi_{i}\left(t_{i, n}\right) \geq \pi \\
\frac{\varphi_{i}\left(t_{i, n+1}\right)+\varphi_{i}\left(t_{i, n}\right)-2 \pi}{4 \pi} \lambda, \varphi_{i}\left(t_{i, n+1}\right)-\varphi_{i}\left(t_{i, n}\right) \leq-\pi
\end{array}\right.
$$

Where $\varphi_{i}\left(t_{i, n}\right)$ is the phase measurement by the $i^{\text {th }}$ antenna at timestamp $t_{i, n}$. Further, the radical speed $\vec{v}_{i}\left(t_{i, n}\right)$ can be obtained:

$$
\left|\vec{v}_{i}\left(t_{i, n}\right)\right|=\frac{\Delta d}{t_{i, n+1}-t_{i, n}}
$$

Actually, the method to estimate the radial speed by measuring the phase values at adjacent time moments can be regarded as a form of measuring Doppler shift to determine the speed of the mobile tag. The relation between radial speed and instant speed can be expressed as:

$$
\left|\vec{v}_{i}\left(t_{i, n}\right)\right|=\left|\vec{v}\left(t_{n}\right)\right| \cos \left(\theta\left(t_{n}\right)-\theta_{i}\left(t_{i, n}\right)\right)
$$

where $\theta\left(t_{n}\right)$ and $\theta_{i}\left(t_{i, n}\right)$ are the direction of vector $\vec{v}\left(t_{n}\right)$ and $\vec{v}_{i}\left(t_{n}\right)$ respectively. The instant velocity can be estimated by utilizing the nonlinear least-squares method:

$$
\min \sum_{i=1}^{N}\left[\left|\vec{v}\left(t_{n}\right)\right| \cos \left(\theta\left(t_{n}\right)-\theta_{i}\left(t_{i, n}\right)\right)-\left|\vec{v}_{i}\left(t_{i, n}\right)\right|\right]^{2}
$$

When every round of antenna schedule ends, a series of radical speeds of the tag can be obtained and the instant speed can be estimated based on (10). Then the trajectory can be updated using (3).

\section{SIMULATION}

In order to verify the performance of our algorithm and our assumption that the trajectory can be approximated by a series of linear segments, we simulate a mobile RFID tag moving along a circular track at a constant speed of $1.5 \mathrm{~m} / \mathrm{s}$, as shown in figure 3.

The radius of the track is $1 \mathrm{~m}$ and the track's center is at $\mathrm{x}=1.5 \mathrm{~m}$ and $\mathrm{y}=1.5 \mathrm{~m}$. Four antennas are located at the four corners of a square with $3 \mathrm{~m}$ length.

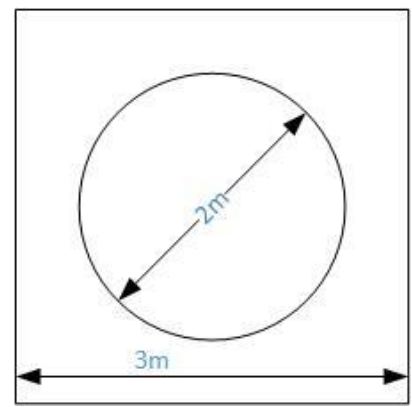

Fig. 3. Simulation of circular track

The tag is interrogated 30 times per second by each antenna. We assume free space propagation and the phase measurements is distorted by white noise. The simulations are carried out in MATLAB.

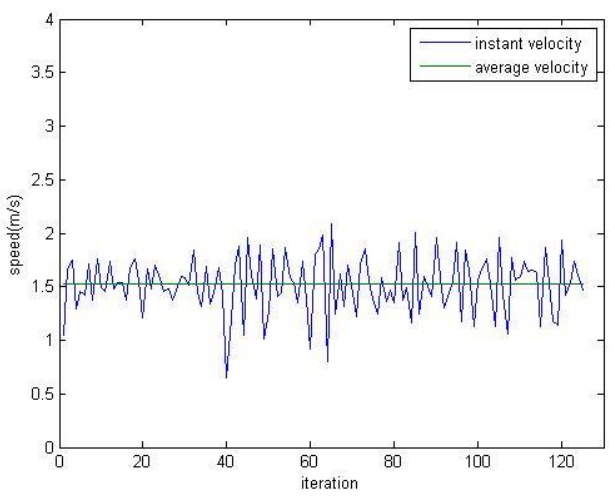

Fig. 4. Instant velocity estimation

After the tag moves one lap anticlockwise, a total of 126 positions are estimated. Based on our approach, we estimate the initial positon using least squares. The starting position is determined at $(2.47 \mathrm{~m}, 1.57 \mathrm{~m})$. Then by leveraging the Doppler shift, the radical speed of the mobile tag at each position is calculated. The instant speed can be derived from the radial speed. As shown in Fig. 4, the estimated average speed is $1.5214 \mathrm{~m} / \mathrm{s}$, which is very close to the real speed.

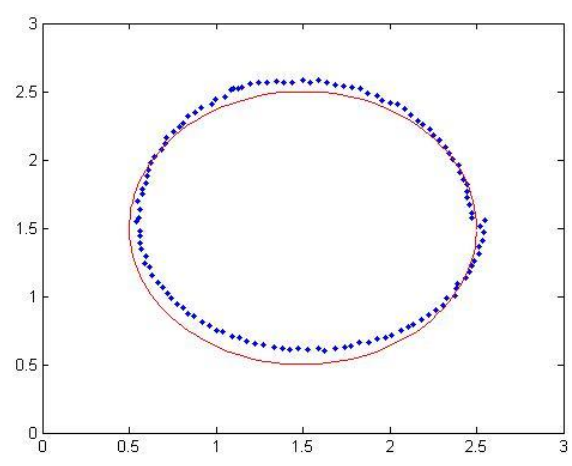

Fig. 5. Trajectory estimation

Because the tag travels only $5 \mathrm{~cm}$ during each time interval, the actual circular track can be approximated by a piecewise linear curve. After the initial position and instant 
velocity have been calculated, the trajectory can be updated recursively. The result is shown in Fig.5. The median error is $0.1027 \mathrm{~m}$, and standard deviation is $0.0154 \mathrm{~m}$. Moreover, the moving direction of the tag is changing continuously and the estimated trajectory using our method still follows the circular track. Therefore, our approximation using linear segments is viable. The performance of our method is demonstrated to be accurate not only in the estimation of instant speed but also the trajectory tracking.

\section{MEASUREMENT}

Measurements were carried out in the knowledge discovery laboratory of NTNU. We adopted a Sirit Infinity 610 reader without any modification of the firmware. The reader is a multi-protocol radio frequency identification system that operates in the $860-960 \mathrm{MHz}$ UHF band. The tag is mounted on a moveable cart and is manually moved along a predefined trajectory marked on the floor, as shown in Fig.6.

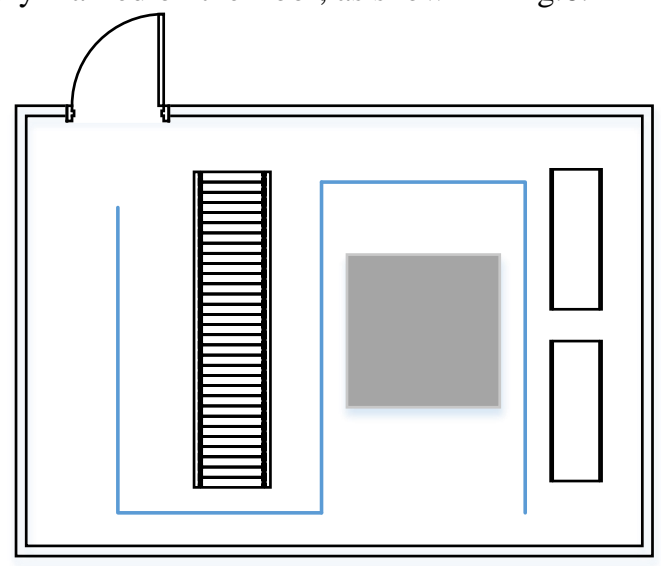

Fig. 6. The trajectory of the true path

Totally, 245 points are estimated during the measurement. The mean accuracy of tracking is $0.1218 \mathrm{~m}$. Generally, the tracking result is acceptable and the calculated trajectory follows along the predefined track. The main discrepancy comes from the corner where the tag's direction changes abruptly. That will result in the calculation error of radical speed. If the transition from one direction to another is connected with arc, the accuracy will improve further.

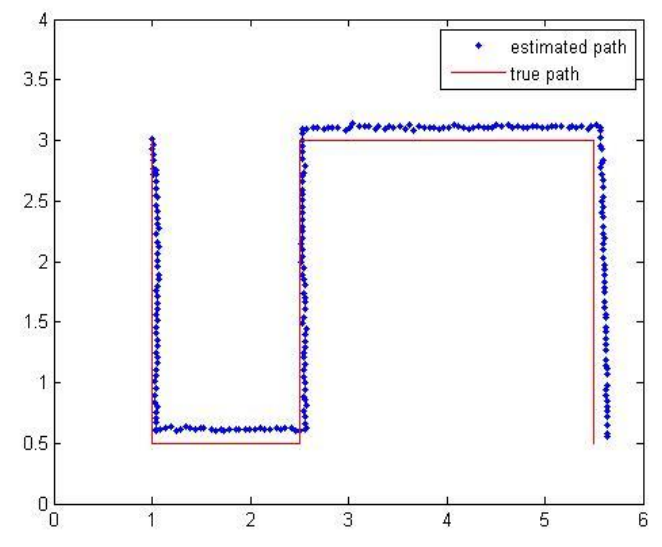

Fig. 7. Experimental results

\section{CONCLUSION}

This paper proposes a novel cost-efficient method for realtime indoor tracking of mobile RFID tag using COTS devices and ensuring the localization accuracy at the same time. The position of the mobile tag can be estimated without priori knowledge of the velocity and trajectory. Because the distance traveled by the tag is far less than half a wavelength during each time interval, it is reasonable to approximate the trajectory into a piecewise linear curve. The movement in each segment can be viewed as linear uniform motion. We estimate the initial position based on RSSI using least squares. Then the radical speed of the mobile tag is calculated by measuring the Doppler shift. The instant speed is fitted using nonlinear least squares. Out method is verified to be able to provide accurate velocity and trajectory estimation.

\section{REFERENCES}

[1] S. Särkkä, V.V. Viikari, M. Huusko, and K. Jaakkola, "Phase-based UHF RFID tracking with nonlinear Kalman filtering and smoothing," IEEE Sensors J., vol. 12, no. 5, pp. 904-910, 2012

[2] H. Zou, B. Huang, X. Lu, H. Jiang, and L. Xie, "A Robust Indoor Positioning System Based on the Procrustes Analysis and Weighted Extreme Learning Machine," IEEE Transactions on Wireless Communications, vol. 15, no. 2, pp. 1252-1266, 2016

[3] A. Bekkali, H. Sanson, and M. Matsumoto, "RFID indoor positioning based on probabilistic RFID map and Kalman filtering," in Third IEEE International Conference on Wireless and Mobile Computing, Networking and Communications (WiMob 2007), 2007, pp. 21-21.

[4] L.M. Ni, Y. Liu, Y.C. Lau, and A.P. Patil, "LANDMARC: indoor location sensing using active RFID," Wireless networks, vol. 10, no. 6, pp. 701-710, 2004

[5] Z. Chen, H. Zou, H. Jiang, Q. Zhu, Y.C. Soh, and L. Xie, "Fusion of WiFi, smartphone sensors and landmarks using the Kalman filter for indoor localization," Sensors, vol. 15, no. 1, pp. 715-732, 2015

[6] S. Mahfouz, F. Mourad-Chehade, P. Honeine, J. Farah, and H. Snoussi, "Target tracking using machine learning and Kalman filter in wireless sensor networks," IEEE Sensors Journal, vol. 14, no. 10, pp. 3715-3725, 2014

[7] A. Buffi, P. Nepa, and F. Lombardini, "A phase-based technique for localization of UHF-RFID tags moving on a conveyor belt: performance analysis and test-case measurements," IEEE Sensors Journal, vol. 15, no. 1, pp. 387-396, 2015

[8] L. Yang, Y. Chen, X. Li, C. Xiao, M. Li, and Y. Liu, "Tagoram: Real-time tracking of mobile RFID tags to high precision using COTS devices," in Proc. 20th annu. Int. Conf. Proceedings of the 20th annual. MobiCom, 2014, pp. 237-248.

[9] sirit, "Application Note for Tag Phase Reporting Technology," sirit Application Note, vol., no., 2009 